\title{
Instrumented Trail-Making Task: Application of Wearable Sensor to Determine Physical Frailty Phenotypes
}

\author{
He Zhou $^{a}$ Javad Razjouyan ${ }^{a, b}$ Debopriyo Halder ${ }^{a, c}$ Anand D. Naik ${ }^{b, d}$ \\ Mark E. Kunik ${ }^{b, d, e ~ B i j a n ~ N a j a f i a ~}$
}

anterdisciplinary Consortium on Advanced Motion Performance (iCAMP), Michael E. DeBakey Department of

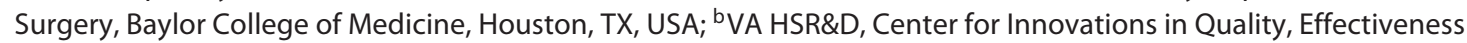
and Safety, Michael E. DeBakey VA Medical Center, Houston, TX, USA; ${ }^{\circ}$ College of Natural Sciences and Mathematics, University of Houston, Houston, TX, USA; ${ }^{d}$ Department of Medicine, Section of Health Services Research, Baylor College of Medicine, Houston, TX, USA; ' $V A$ South Central Mental Illness Research, Education and Clinical Center, Houston, TX, USA

\section{Keywords}

Frailty $\cdot$ Instrumented trail-making task $\cdot$ Frailty phenotype .

Gait · Wearable $\cdot$ Virtual-reality $\cdot$ Cognitive-motor test

\begin{abstract}
Background: The physical frailty assessment tools that are currently available are often time consuming to use with limited feasibility. Objective: To address these limitations, an instrumented trail-making task (iTMT) platform was developed using wearable technology to automate quantification of frailty phenotypes without the need of a frailty walking test. Methods: Sixty-one older adults (age $=72.8 \pm 9.9$ years, body mass index $[\mathrm{BMI}]=27.4 \pm 4.9 \mathrm{~kg} / \mathrm{m}^{2}$ ) were recruited. According to the Fried Frailty Criteria, 39\% of participants were determined as robust and $61 \%$ as non-robust (pre-frail or frail). In addition, 17 young subjects (age $=29.0 \pm 7.2$ years, $\mathrm{BMI}=26.2 \pm 4.6 \mathrm{~kg} / \mathrm{m}^{2}$ ) were recruited to determine the healthy benchmark. The ITMT included reaching 5 indexed circles (including numbers 1-to-3 and letters A\&B placed in random orders), which virtually appeared on a computerscreen, by rotating one's ankle-joint while standing. By using
\end{abstract}

\section{KARGER}

๑) 2018 S. Karger AG, Basel

E-Mail karger@karger.com

www.karger.com/ger an ankle-worn inertial sensor, 3D ankle-rotation was estimated and mapped into navigation of a computer-cursor in realtime $(100 \mathrm{~Hz})$, allowing subjects to navigate the computercursor to perform the iTMT. The ankle-sensor was also used for quantifying ankle-rotation velocity (representing slowness), its decline during the test (representing exhaustion), and ankle-velocity variability (representing movement inefficiency), as well as the power (representing weakness) generated during the test. Comparative assessments included Fried frailty phenotypes and gait assessment. Results: All subjects were able to complete the iTMT, with an average completion time of $125 \pm 85 \mathrm{~s}$. The iTMT-derived parameters were able to identify the presence and absence of slowness, exhaustion, weakness, and inactivity phenotypes (Cohen's $d$ effect size $=0.90-1.40$ ). The iTMT Velocity was significantly different between groups ( $d=0.62-1.47$ ). Significant correlation was observed between the iTMT Velocity and gait speed $(r=0.684 p<0.001)$. The iTMT-derived parameters and age together enabled significant distinguishing of non-robust cases with area under curve of 0.834 , sensitivity of $83 \%$, and specificity of $67 \%$. Conclusion: This study demonstrated a non-gait-based wearable platform to objectively quantify 
frailty phenotypes and determine physical frailty, using a quick and practical test. This platform may address the hurdles of conventional physical frailty phenotypes methods by replacing the conventional frailty walking test with an automated and objective process that reduces the time of assessment and is more practical for those with mobility limitations.

(c) 2018 S. Karger AG, Basel

\section{Introduction}

Frailty is a geriatric syndrome that increases an olderadult's vulnerability to falls, hospitalization, long-term care placement, and mortality [1]. Recent epidemiologic studies show that, in the United States, there are around 3.6 million people with frailty [2]. The total cost related to frailty each year is approximately USD 18.5 billion or $15 \%$ of the total healthcare expenditure in the United States [3]. Early diagnosis of frailty can help patients and physicians make more informed medical decisions of modifiable risk factors to reduce postoperative adverse or poor functional outcomes [4]. Although several studies have suggested that frailty is an irreversible process, it has been hypothesized that the early detection of frailty stages may provide a window of opportunity for timely preventive or therapeutic interventions, which may delay the progression of frailty and even reverse it [5]. Thus, a practical and quick tool to determine frailty stages irrespective of setting (e.g., at home or clinic) is desperately needed.

Currently, there is no gold standard for diagnosing frailty and "old age" itself does not define frailty [6]. One of the most widely accepted models to assess the frailty syndrome was proposed by Fried's group in 2001 (Fried Frailty Criteria, FFC) [1]. Five different phenotypes (slowness, weakness, exhaustion, inactivity, and shrinking or involuntary weight loss) were suggested as criteria to determine different frailty stages. Subjects who are positive for 1 or 2 phenotypes are considered pre-frail, while those with 3 or more positive phenotypes are considered frail. Subjects who are negative for all 5 phenotypes are considered robust. The FFC has been proved to be accurate for identifying older adults with low resilience and high vulnerability, and poor health outcomes can be predicted with it independently of comorbidities. However, it has limited feasibility and reliability in routine clinical applications [7]. In addition, it is not sensitive to track changes in frailty stages over time [8]. Specifically, certain self-reported criteria used in the FFC are subjective and prone to self-report bias [9]. Furthermore, one of the most important criteria of the FFC is slowness, assessed by a 15 -foot $(\sim 5 \mathrm{~m})$ walking test. This test could be challenging to administer in busy clinics and among frail patients. There are some other methods for frailty assessment $[9,10]$. Unfortunately, all of these methods share similar limitations as the FFC.

Recent advances in designing wearable, virtual-reality, and interactive-interface technologies have created new opportunities to design practical and time-efficient tools, which provide objective metrics to quantify motor functional performance, identify cognitive impairment, and track health status, irrespective of setting and across disciplines [11-15]. Recently we designed a non-gait-based cognitive-motor assessment tool named the instrumented trail-making task (iTMT). In our previous studies [16, 17], we have demonstrated that the iTMT is sensitive enough to determine cognitive function in older adults. To continue these efforts, in the current study, we examined whether the iTMT is able to distinguish different frailty stages (robust, pre-frail, and frail), as determined by the FFC. In addition, we examined whether the iTMTderived parameters could describe physical frailty phenotypes. Our basic premise was that older adults with frailty or pre-frailty will have poorer performance, as measured by the iTMT, than robust or young subjects.

\section{Methods}

\section{Study Population}

Sixty-one older adults were recruited from specialized outpatient clinics (e.g., Cancer Center, Alzheimer's Disease and Memory Disorders Center, Geriatric Clinic, Endocrine Surgery Clinic, etc.). To be eligible, subjects had to be ambulatory, aged at least 60 , and willing to participate in this study. Subjects were excluded if they were unable to walk $20 \mathrm{~m}$ with or without walking assistance; had significant visual problems that limited their ability to interact with a computer-screen with or without visual correction; had lower-extremity problems that limited their ability to perform ankle-rotation; or had severe balance impairment that limited their ability to independently stand for at least $1 \mathrm{~min}$. Those who could stand behind a chair and perform the iTMT test by holding the chair were not excluded. To compare the iTMT results between young and older adults, as well as to determine a healthy benchmark, 17 young ambulatory subjects with ages ranging from 20 to 35 years were also recruited. This study was approved by the local IRBs.

\section{Clinical and Motor Performance Measurements}

We first applied the FFC to all older subjects. According to the FFC, 24 subjects (39\%) were classified as robust, 29 subjects (48\%) were classified as pre-frail, and 8 subjects (13\%) were classified as frail. Since the sample size of the frail group was small, we further combined the pre-frails and frails as a non-robust group. Subjects' demographics, including age, gender, weight, height, body mass 
Fig. 1. The iTMT included standing in front of a standard computer and performing a series of virtual trail-making tests by rotating ankle joint. Subjects were allowed to hold a chair or table for support if needed. A shin-worn sensor enabled measuring $3 \mathrm{D}$ rotation of ankle joint and mapped it into movement of a computer-cursor in real-time (sample frequency of $100 \mathrm{~Hz}$ ). This allowed a smooth navigation of the computer-cursor by rotation of ankle joint. The iTMT reaching tasks include bringing the cursor back and forth to 5 targets indexed with numbers (1-3) and letters $(A, B)$ in the sequential number-letter order (i.e., 1, A, 2, $B$, and 3 ). Audio and visual feedback were provided to assist accurate execution of the tasks in the correct order.

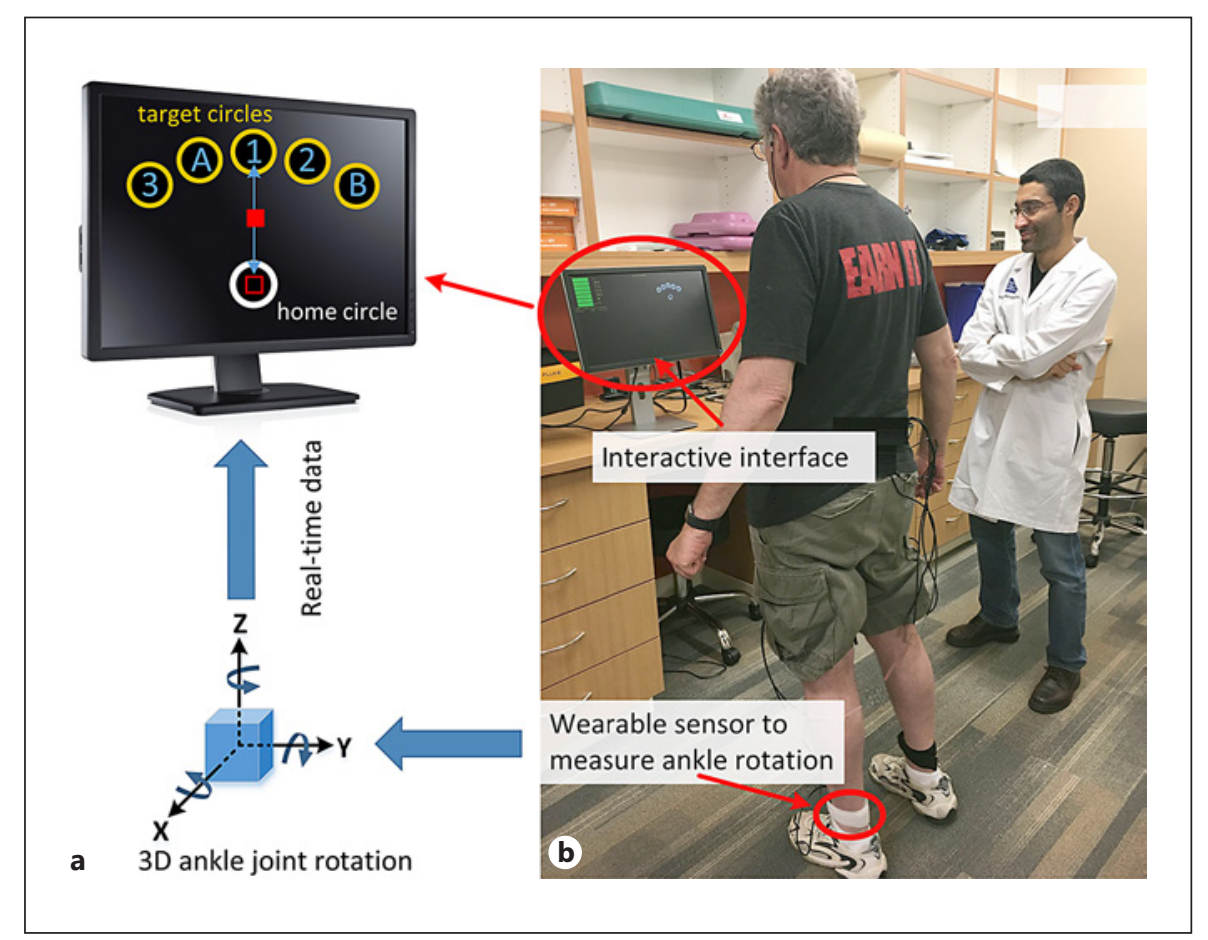

index, daily number of prescription medicines, daily number of over-the-counter medicines, use of walking assistance, and fall history were collected. All subjects underwent clinical assessments, including Falls Self-Efficacy Scale (FES-I) and Center for Epidemiologic Studies Depression (CES-D) scale. The FES-I and cutoff score suggested by Delbaere et al. [18] was used to identify subjects with high concern about falling. The CES-D short-version scale was used to measure self-reported depression symptoms. A cutoff of CES-D score of 16 or greater was used to identify subjects with depression [19]. Gait performance was measured using wearable sensors (LegSys ${ }^{\mathrm{TM}}$, BioSensics, MA, USA) attached to both left and right lower legs. Subjects were asked to walk with their habitual gait speed for $20 \mathrm{~m}$ without any distraction. Using a validated model (double inverse pendulum model representing motion around ankle and knee joint), we calculated gait speed [20,21].

\section{iTMT Platform Design}

We designed the iTMT platform (Fig. 1) based on a single inertial wearable sensor (LEGSys ${ }^{\mathrm{TM}}$, BioSensics, MA, USA), which includes a triaxial accelerometer, a triaxial gyroscope, and a triaxial magnetometer. This configuration enabled the estimation of $3 \mathrm{D}$ joint angles [21]. Sensor data were acquired and transmitted at $100 \mathrm{~Hz}$ frequency for real-time feedback.

Using an elastic strap, the sensor was attached to the subject's lower shin of the dominant leg (Fig. 1b). The sensor enabled tracking ankle-rotation in 3D. The data were transmitted to an interactive interface installed on a standard computer (Fig. 1b). By rotating the ankle, the subject could navigate a cursor on the computerscreen from a home circle to target circles appearing on the same screen. In summary, the sensor provided quaternion components of ankle-rotation with a sample frequency of $100 \mathrm{~Hz}$. Using the method described in our previous studies [22, 23], we calibrated the sensor at the beginning of the test, when the subject was in the upright position and resting (no movement). The calibration process corrected the misalignment of the sensor with respect to gravity, bringing the cursor to the home circle (Fig. 1a) at the beginning of each iTMT trial. In addition, it saved the coordinates of the body landmarks (reference axes), representing frontal, sagittal, and transverse planes for the subject. The code updated reference axes at the beginning of each iTMT trial to reduce the effect of potential drift. All subsequent quaternion components during movement were subsequently converted to Euler angles with respect to the reference axes. These angles were used to describe a sequence of 3 rotations, determining the orientation of the shank segment in 3 dimensions, including yaw, pitch, and roll. The yaw and pitch components were used to estimate components of " $\mathrm{X}$ " (horizontal movement) and "Y" (vertical movement) of the cursor in real time. The estimated $\mathrm{X}$ and $\mathrm{Y}$ cursor positions were updated on the screen with a sample frequency of $100 \mathrm{~Hz}$. The cursor velocity, representing the ankle-rotation velocity, in directions of $\mathrm{X}$ and $\mathrm{Y}$ were then estimated from the change of the position of the cursor. A code was developed to identify the "start" and "end" points for each iTMT trial. The "start" point was determined when the cursor was inside of home circle, and cursor velocity exceeded a predefined threshold (initiation of movement). The "end" point was determined when the cursor reached one of the target circles, and cursor velocity was below a predefined threshold (cursor stopped in the target circle). In our previous study, we demonstrated that all calculation processes and visualization of the cursor could be executed in real time with a sample frequency of $100 \mathrm{~Hz}$ [24]. At the end of the iTMT test, the platform recorded all sensor data and the iTMT key variables for the post-processing phase. 
Table 1. Definition of the iTMT terms

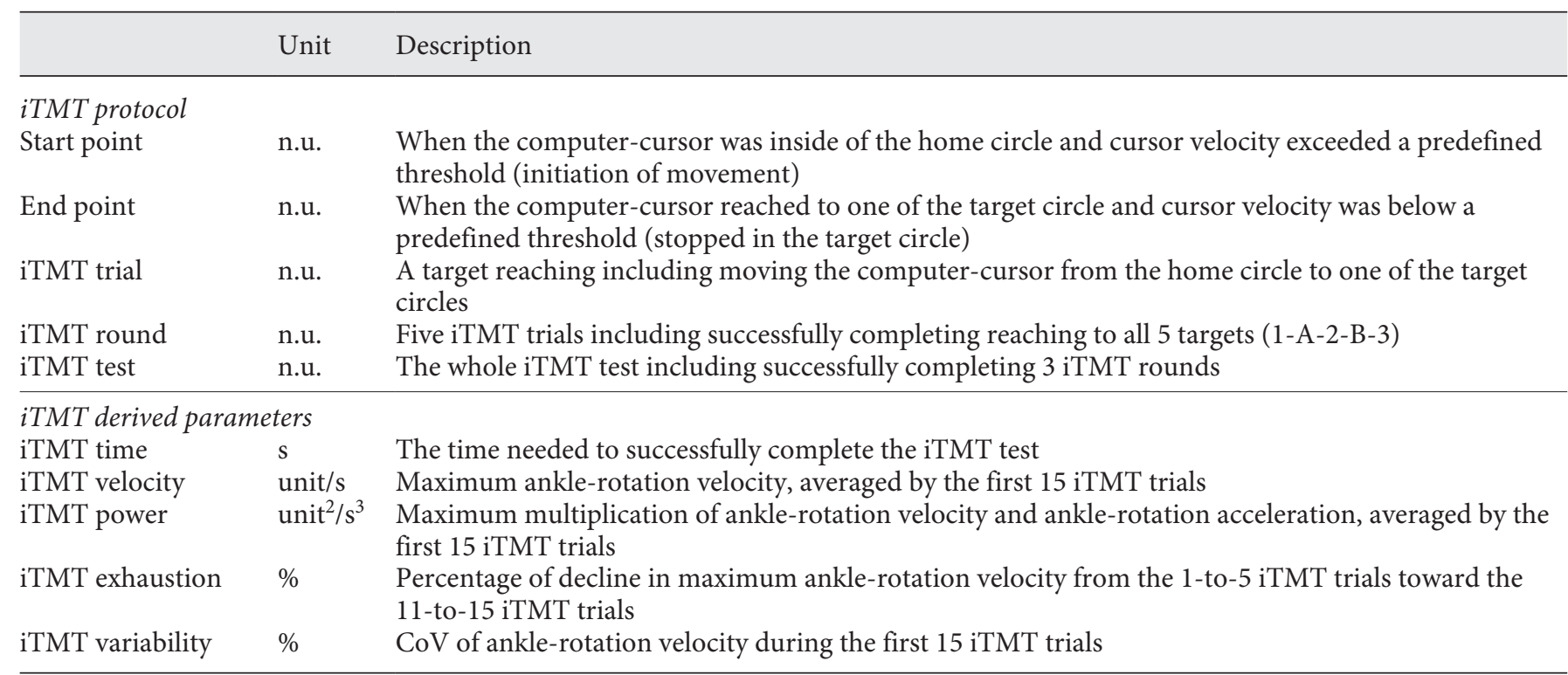

iTMT, instrumented trail-making task; CoV, coefficient of variation; n.u., no unit.

Definitions of the iTMT protocol and iTMT-derived parameters are summarized in Table 1 and will be described in the following sections.

\section{iTMT Protocol}

The subject was instructed to stand in front of the computerscreen, while wearing the sensor on the lower shin of the dominant leg (Fig. 1b). For safety purposes, a research coordinator was in the room supervising the iTMT test at all times. Subjects were given the option of using the support of either a sturdy chair or a table placed in front of them to maintain balance during anklerotation, which required weight-shifting tasks. Before starting the iTMT, the research coordinator described the protocol to the subject. After starting the iTMT test, the research coordinator did not provide any further guidance; only the interactive interface provided the necessary guidance and instructions, as described by the following.

For trail-making, the subject needed to stand upright (always in double stance) and move the hip in the anterior-posterior direction to generate dorsiflexion/plantarflexion at the ankle without lifting the heels or toes (Fig. 2a). The subject navigated the cursor to correct targets in a certain order, by rotating the ankle joint, defined as an ankle-rotation task [24].

In the iTMT test, 6 circles appeared on the screen -1 home circle in white and 5 target circles in yellow (Fig. 1a). The target circles were located in a fanwise position in front of the home circle. Each target circle had a number ("1", "2", or " 3 ") or letter ("A" or "B") located in the center (the order of numbers and letters was randomized). At the beginning of the iTMT, the position of the cursor was automatically calibrated to the center of the home circle as described earlier. By rotating the ankle joint, the subject navigated the cursor from the home circle to the center of target circles with numbers and letters alternately, defined as the iTMT trial. To be specific, the subject navigated the cursor from the home circle to the center of the first target circle (with number " 1 " inside, iTMT trial \#1). Then the subject navigated the cursor back to the home circle and went to the second target circle (with letter " $A$ " inside, iTMT trial \#2), and came back to the home circle and went to the third target circle (with number "2" inside, iTMT trial \#3), and went on. When the cursor stopped at the center of the last target circle (with number " 3 " inside, iTMT trial \#5), 1 round of the test, defined as the iTMT round, was completed. The whole iTMT test contains 3 continuous rounds. For the iTMT test, besides performing the motor task, the subject also needed to observe and figure out the correct location of the next target and remember to switch between number and letter sequences (cognitive tasks associated) $[16,17]$. If the subject navigated the cursor to the correct target circle, the border of the target circle would turn red; and the target circle would explode with a rewarding sound. If the subject navigated the cursor to a wrong target circle, a visual and audio feedback indicating a mistake would be played. When that occurred, the subject had to go back to the home circle and continue the iTMT from where he or she made the previous mistake. If the subject made 3 consecutive mistakes, a visual cue (flashing of correct target circle) would appear to guide him/her to correct the sequence.

\section{iTMT-Derived Parameters}

Several measurable parameters were derived from the sensor data, including iTMT time, iTMT Velocity, iTMT Power, iTMT Exhaustion, and iTMT Variability (Table 1). The iTMT time was defined as the time needed to successfully complete the iTMT test and was shown to be correlated with cognitive function $[16,17]$. In this study, we explored other iTMT-derived parameters. 


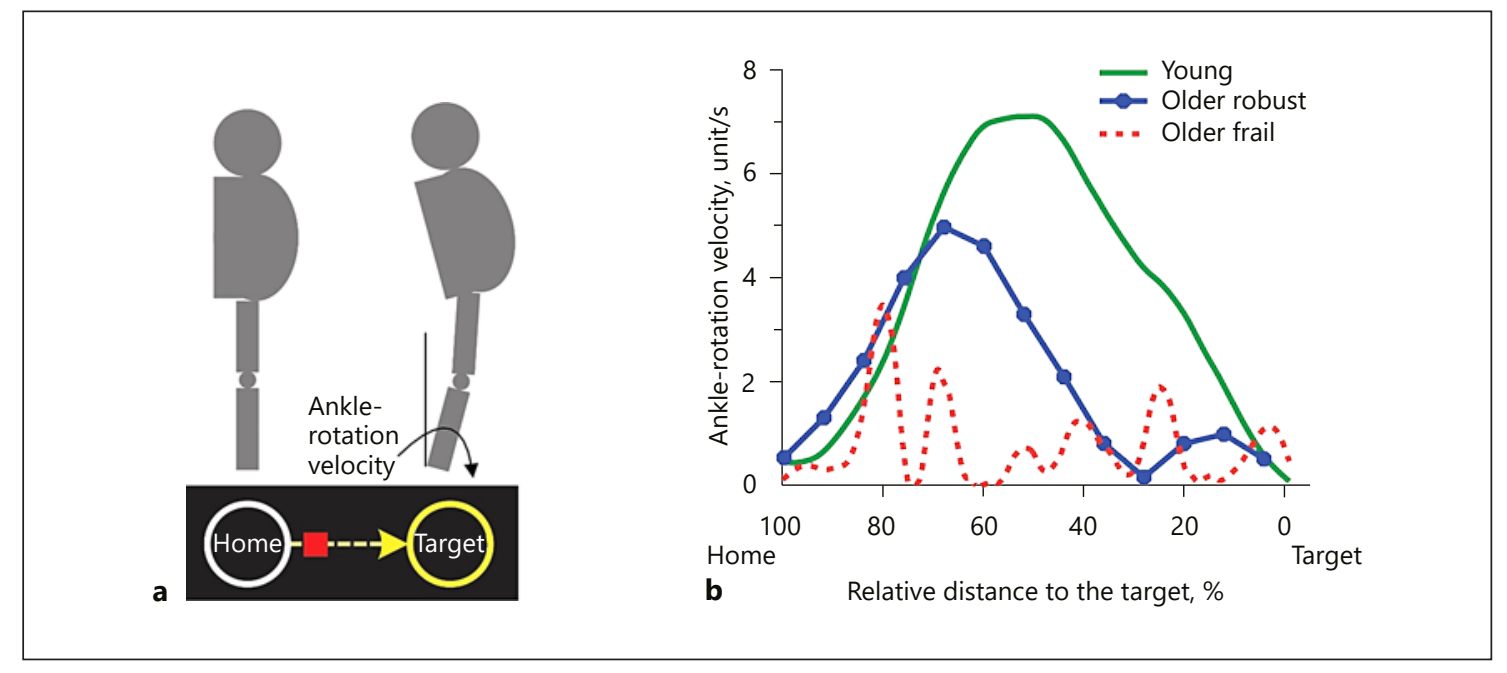

Fig. 2. a For trail-making, the subject needed to stand upright (always in double stance) and move the hip in the AP direction in order to generate dorsiflexion/plantarflexion at the ankle without lifting heels or toes. The rotation of ankle was mapped into movement of a computer-cursor. A reaching task is defined by navigating the cursor (by rotating ankle joint) from a home circle and stopping the cursor at the middle of a target circle. If the subject achieved to navigate rapidly $(<1 \mathrm{~s})$ and accurately (in the middle

During the post-processing phase, we used data recorded from sample-to-sample cursor velocity during each iTMT trial to estimate the ankle-rotation velocity curve (Fig. 2b). In summary, the norm of recorded cursor velocities in directions of $\mathrm{X}$ and $\mathrm{Y}$ was estimated. Next, a low pass filter using wavelet transfer (mother wavelet: $\mathrm{db} 5$, cut of frequency: $6.25 \mathrm{~Hz}$ ) was applied. The maximum value of ankle-rotation velocity for each iTMT trial was estimated. The iTMT Velocity was defined as the average of maximum ankle-rotation velocities across the first 15 iTMT trials. This was done for a fair comparison between subjects, considering that some subjects (in particular, those with cognitive impairment) may have more than 15 iTMT trials (due to mistakes they made) to complete the iTMT test. To estimate the power, ankle-rotation velocity and acceleration were multiplied for each iTMT trial. The ankle-rotation acceleration was estimated with a single derivation of ankle-rotation velocity [25]. The iTMT Power was calculated as the maximum power during the iTMT test, averaged for the first 15 iTMT trials. The iTMT Exhaustion was calculated as the percentage of decline in maximum ankle-rotation velocity from the 1-to-5 iTMT trials toward the 11-to-15 iTMT trials. If the maximum ankle-rotation velocity was higher in the 11-to-15 iTMT trials, the iTMT Exhaustion was considered 0\%. The iTMT variability was calculated as the coefficient of variation of ankle-rotation velocity during the first 15 iTMT trials.

Figure $2 \mathrm{~b}$ illustrates patterns of ankle-rotation velocity during a single trail-making, for a typical young subject (green solid curve), older robust subject (blue solid curve with filled circles), and older frail subject (dash-curve in red). At the beginning of the target reaching, the typical young subject first accelerated the ankle-rotation velocity to navigate the cursor on the computer- of the circle) the cursor from the home circle to the correct target circle, the target would explode with a rewarding sound. Different visual and audio feedback were provided to encourage and assist successful completion of the iTMT. b Ankle-rotation velocity for a typical young subject (green solid curve), a typical older robust subject (blue solid curve with filled circles), and a typical frail subject (dash-curve in red).

screen to the target. When the subject reached the maximum ankle-rotation velocity, he or she started to decelerate to achieve zero ankle-rotation velocity for stopping the cursor at the center of the target. For this typical young subject, the ankle-rotation velocity curve had only 1 peak, with a relatively large magnitude (Fig. 2b). For the typical older robust subject, he or she also had a relatively smooth acceleration and deceleration of ankle-rotation velocity. However, the peak velocity was lower than that of the typical young subject. For the typical older frail subject, multiple ankle-rotation velocity peaks could be observed from Figure $2 \mathrm{~b}$, indicating large variability. The peak velocity was lower even when compared to the typical older robust subject.

\section{Statistical Analysis}

All continuous data were presented as mean \pm SD. All categorical data were expressed as count (percentage). The Shapiro-Wilk test was applied for testing normality of data. Analysis of variance was used for comparing the iTMT-derived parameters between different groups and between the presence and absence of each frailty phenotype. Fisher's least significant difference-based post hoc test was performed for pairwise comparison to explore significant main effects or interactions. The effect size to discriminate between groups was estimated using Cohen's $d$ effect size and represented as $d$ in the Results section. Values ranging from 0.20 to 0.49 indicate small effects, and values between 0.50 and 0.79 indicate medium effects. Values ranging from 0.80 to 1.29 indicate large effects, and values above 1.30 indicate very large effects. Values less than 0.20 are considered having no noticeable effect [26]. The Pearson correlation coefficient was used to evaluate the degree of agreement between the iTMT Velocity and conventional gait 
Table 2. General characteristics of the study population

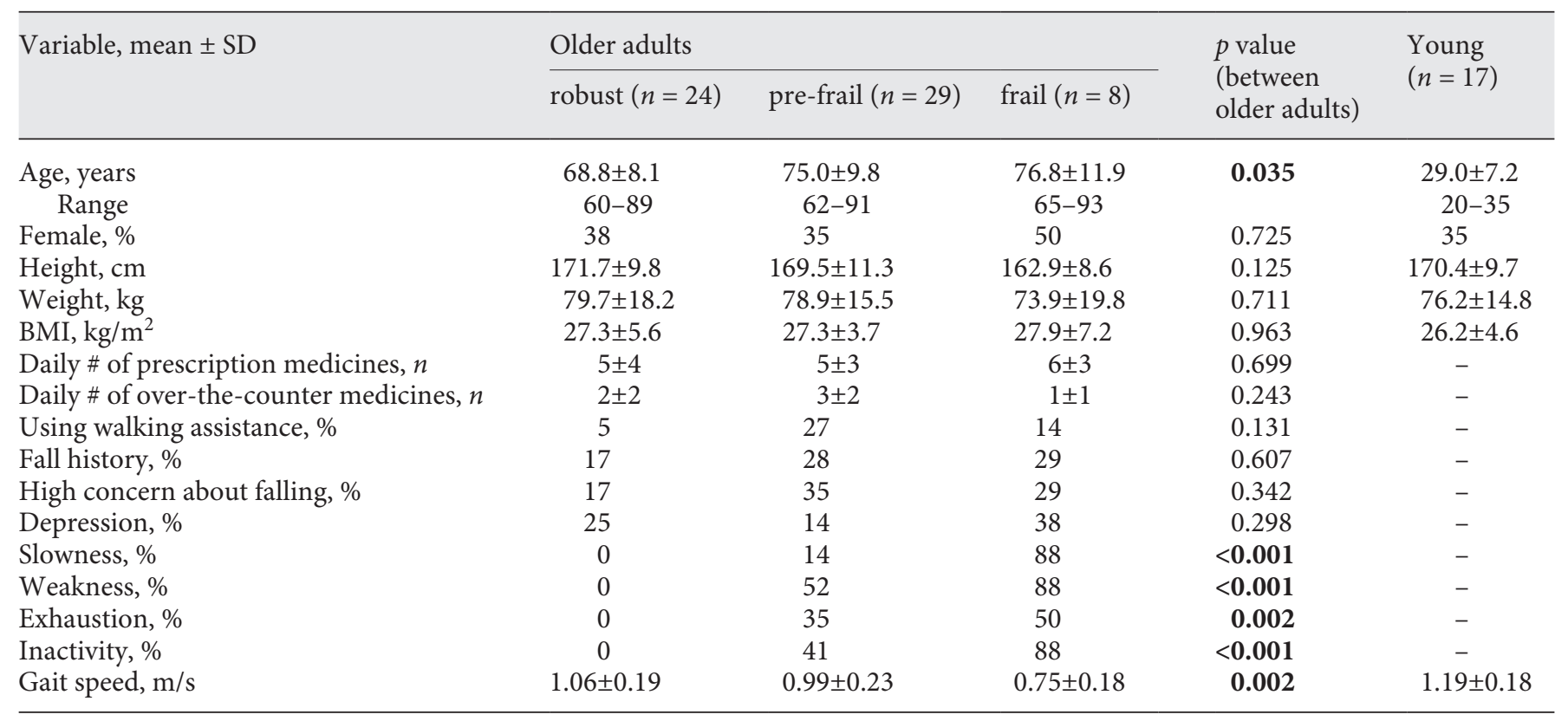

Significant difference between groups is indicated in bold. BMI, body mass index.

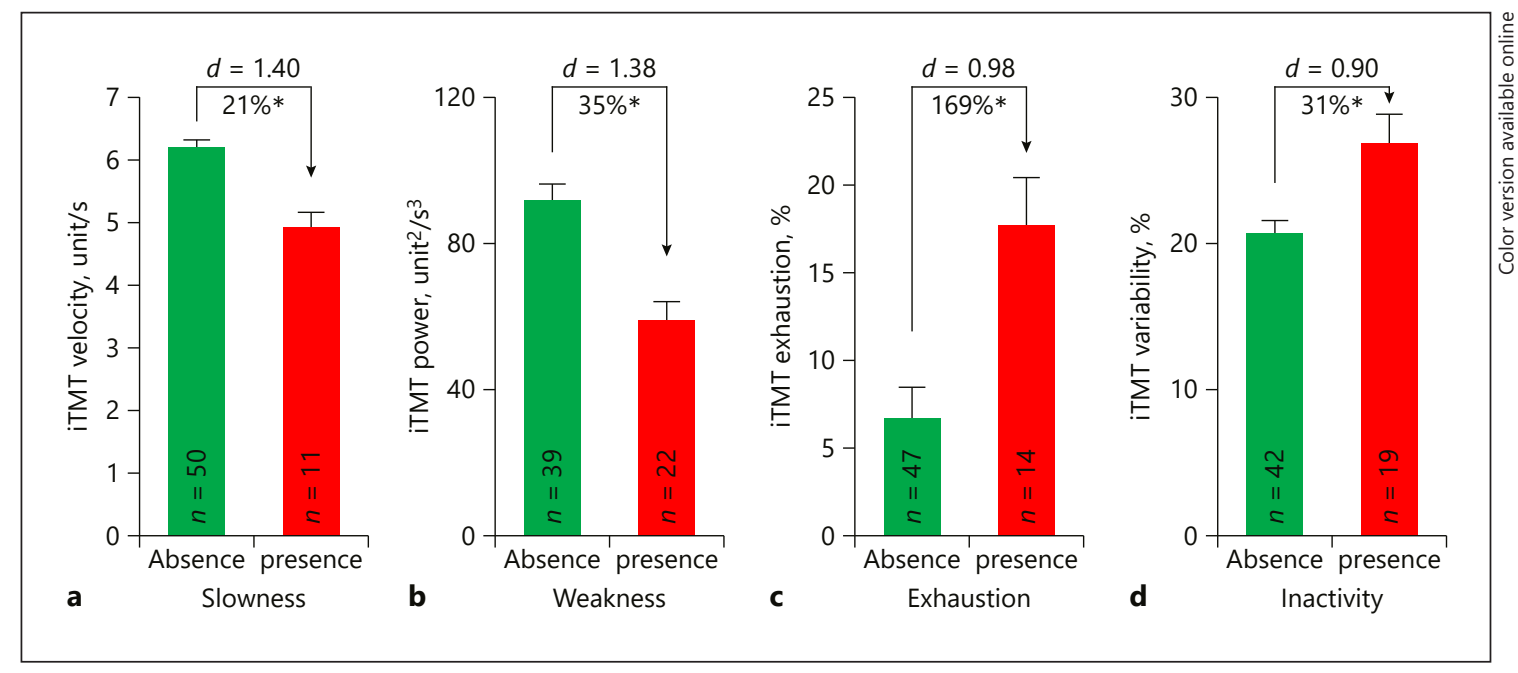

Fig. 3. The instrumented trail-making task (iTMT) derived parameters enabled significant discrimination between the presence and absence of each frailty phenotype as determined by the FCC, including slowness (a), weakness (b), exhaustion (c), and inactivity (d). Error bars represent the standard errors. " $n$ " denotes number of subjects per group. ${ }^{*}$ denotes when the between group comparison achieved a statistically significant level $(p<0.050)$.

speed. Logistic regression analysis was employed to examine the relationship between each study variable and frailty. First, univariate logistic regression was employed to investigate the relationship of the test variables using "non-robust/robust" as the dependent variable. This strategy reflects the exploratory character of the study. The OR was calculated for each explanatory variable. Sec- ond, stepwise multivariate logistic regression, using variables found with $p<0.20$ in the univariate analysis, was performed to investigate the independent effects of variables in predicting frailty. To examine whether the iTMT-derived parameters may yield similar results as gait speed, we used 2 different models. Variables in one of the models (Model 2) included gait speed and other vari- 
Table 3. Between-group comparisons for gait speed and the iTMT-derived parameters

\begin{tabular}{|c|c|c|c|c|c|c|c|c|c|c|c|c|}
\hline & \multicolumn{2}{|l|}{ Older adults } & \multirow{2}{*}{$\begin{array}{l}\text { Young } \\
(n=17)\end{array}$} & \multicolumn{3}{|c|}{ Non-robust vs. robust } & \multicolumn{3}{|c|}{ Robust vs. young } & \multicolumn{3}{|c|}{ Non-robust vs. young } \\
\hline & $\begin{array}{l}\text { robust } \\
(n=24)\end{array}$ & $\begin{array}{l}\text { non-robust } \\
(n=37)\end{array}$ & & $p$ value & $d$ & $95 \% \mathrm{CI}$ & $p$ value & $d$ & $95 \% \mathrm{CI}$ & $p$ value & $d$ & $95 \% \mathrm{CI}$ \\
\hline Gait Speed, m/s & $1.06 \pm 0.19$ & $0.94 \pm 0.24$ & $1.19 \pm 0.18$ & 0.032 & 0.56 & 0.01 to 0.24 & 0.050 & 0.71 & 0.01 to 0.27 & $<0.001$ & 1.21 & 0.13 to 0.38 \\
\hline iTMT Velocity, unit/s & $6.31 \pm 0.98$ & $5.67 \pm 1.09$ & $7.30 \pm 1.13$ & 0.025 & 0.62 & 0.08 to 1.20 & 0.005 & 0.94 & 0.32 to 1.66 & $<0.001$ & 1.47 & 1.01 to 2.25 \\
\hline iTMT Power, unit ${ }^{2} / \mathrm{s}^{3}$ & $90.56 \pm 26.73$ & $73.70 \pm 28.47$ & $113.81 \pm 39.94$ & 0.040 & 0.61 & 0.78 to 32.94 & 0.020 & 0.68 & 3.81 to 42.70 & $<0.001$ & 1.16 & 22.14 to 58.09 \\
\hline iTMT Exhaustion, \% & $8.23 \pm 15.19$ & $9.41 \pm 10.58$ & $4.60 \pm 6.68$ & 0.698 & 0.09 & -7.23 to 4.86 & 0.325 & 0.31 & -10.94 to 3.68 & 0.160 & 0.54 & -11.57 to 1.94 \\
\hline iTMT Variability, \% & $20.92 \pm 4.94$ & $23.05 \pm 7.84$ & $17.15 \pm 6.99$ & 0.241 & 0.33 & -5.73 to 1.46 & 0.088 & 0.62 & -8.12 to 0.58 & 0.005 & 0.79 & 1.88 to 9.92 \\
\hline
\end{tabular}

The older adults non-robust group included pre-frail and frail subjects. Significant difference between groups is indicated in bold. Effect sizes were calculated as Cohen's $d$. iTMT, instrumented trail-making task.

ables found with $p<0.20$ in the univariate analysis, except the iTMT-derived parameters. In Model 3, we replaced gait speed in Model 2 by the iTMT-derived parameters. In Model 1 (reference model), we used all variables found with $p<0.20$ in the univariate analysis, except gait speed and the iTMT-derived parameters. The receiver operating characteristic curve and area under curve (AUC) were calculated for different frailty-prediction models. A 2 -sided $p<0.050$ was considered to be statistically significant. All statistical analyses were performed using IBM SPSS Statistics 24 (IBM, IL, USA).

\section{Results}

All subjects completed the iTMT test, with an average completion time of $125 \pm 85$ s. None of the subjects stopped or were overtaxed during the test, indicating high feasibility of the test. No adverse events, including loss of balance, were observed during the iTMT test.

Table 2 summarizes demographic and clinical data. The older subjects' ages ranged from 60 to 93 . No between-group difference was observed for older subjects' gender, height, weight, body mass index, daily number of prescription medicines, daily number of over-the-counter medicines, and prevalence of depression $(p>0.050)$. However, as expected, the average age of the robust group was significantly younger than that of the pre-frail and frail groups $(p=0.035)$. The pre-frail and frail groups had higher prevalence of using of walking assistance, fall history, and high concern about falling than the robust group. However, the differences did not reach statistical significance. For individual frailty phenotypes, as expected, the frail group had significantly higher prevalence of presence than the pre-frail group for every individual phenotype. For the gait assessment, with progression in frailty, a significant decline of gait speed was observed.

Results suggested that the iTMT Velocity, iTMT Power, iTMT Exhaustion, and iTMT Variability enable sig-

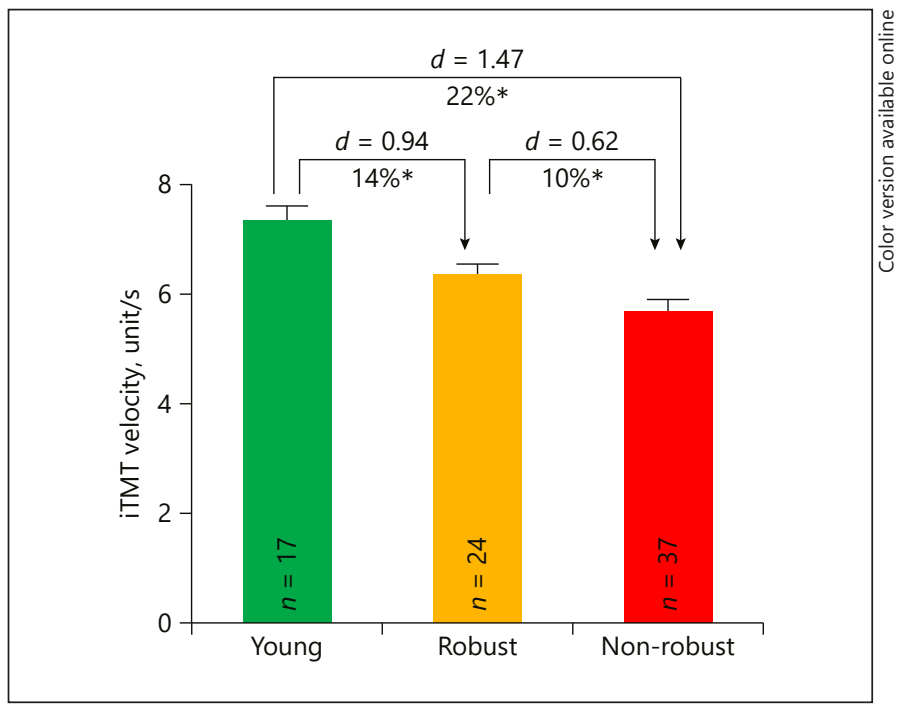

Fig. 4. Average of the instrumented trail-making task (iTMT) Velocity for young, older robust, and older non-robust groups. Error bars represent the standard errors. " $n$ " denotes number of subjects per group. ${ }^{*}$ denotes when the pairwise group comparison achieved a statistically significant level $(p<0.050)$.

nificant discrimination between the presence and absence, as determined by the FFC, of slowness, weakness, exhaustion, and inactivity phenotypes respectively (Fig. 3). In summary, the iTMT Velocity distinguished between the presence and absence of slowness with very large effect size $(d=1.40, p<0.001$, Fig. 3a). Similarly, the iTMT Power determined the presence of weakness $(d=$ $1.38, p<0.001$, Fig. 3b), the iTMT Exhaustion determined the presence of exhaustion $(d=0.98, p=0.003$, Fig. $3 c)$, and the iTMT Variability determined the presence of inactivity $(d=0.90, p<0.001$, Fig. $3 d)$, all with large-tovery-large effect sizes.

Table 3 summarizes gait speed and the iTMT-derived parameters for older robust subjects, older non-robust 
Table 4. Results of univariate logistic regression

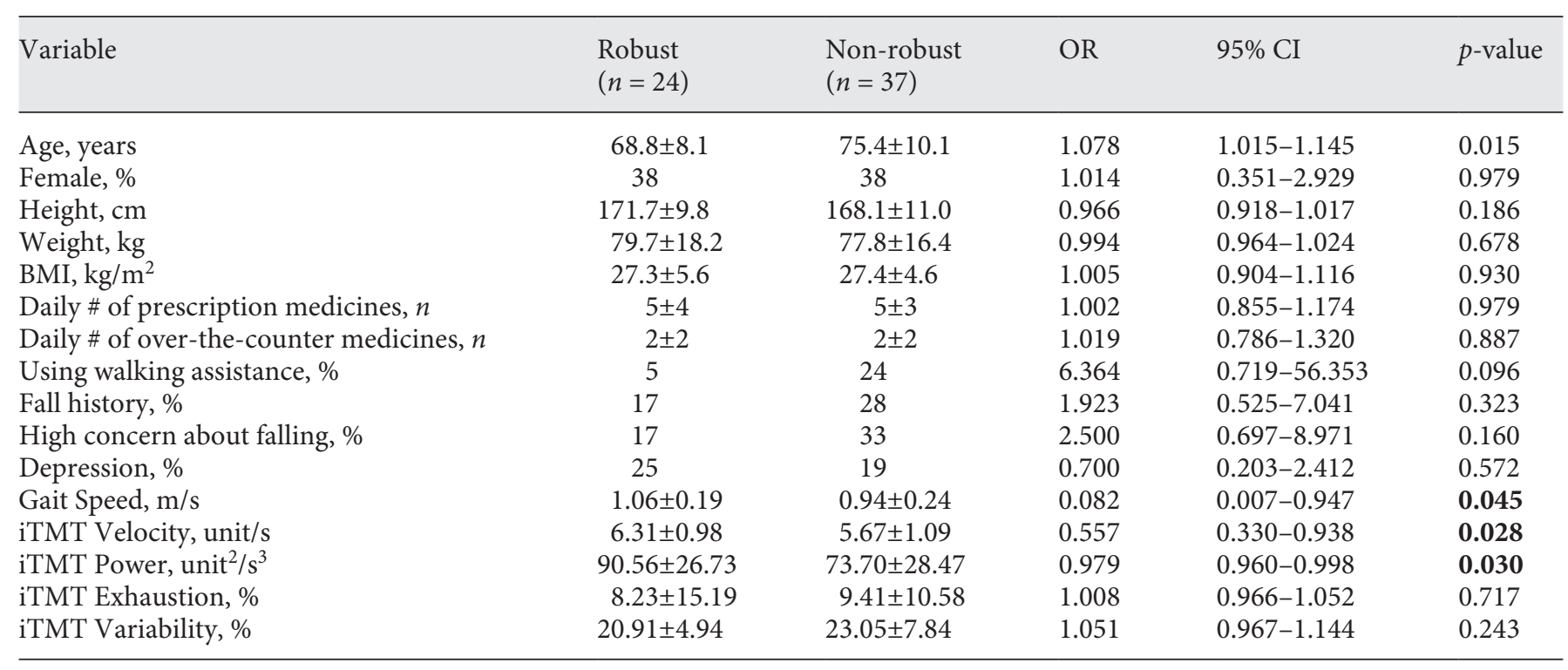

Significant difference between groups is indicated in bold.

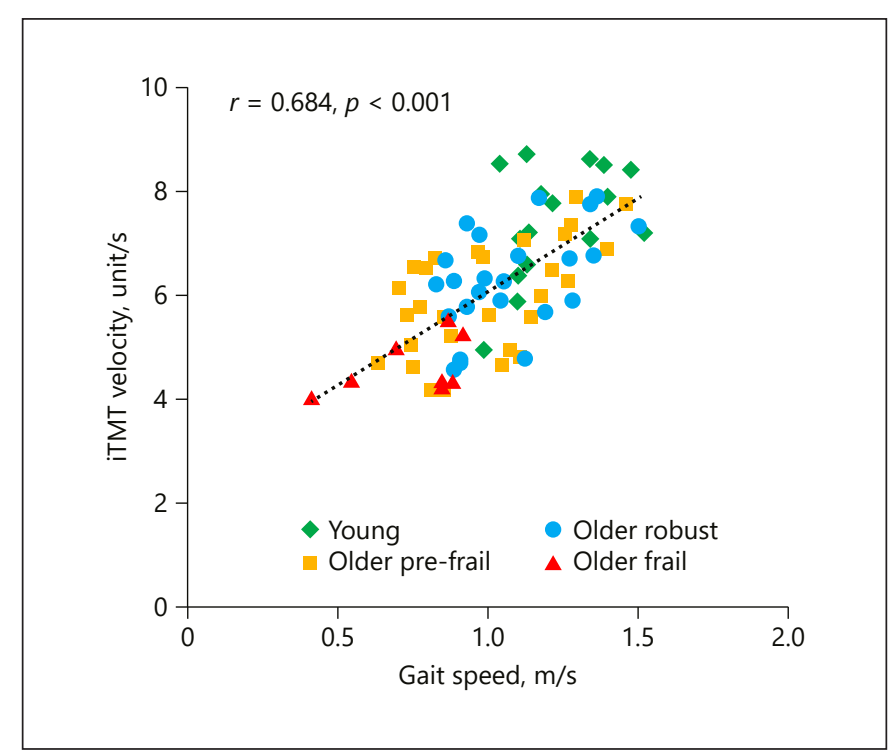

Fig. 5. A significant correlation was observed between the instrumented trail-making task (iTMT) Velocity and over ground gait speed.

subjects, and young subjects. The older non-robust group had poorer performance for gait speed and all of the parameters, as measured by the iTMT, than the robust and young subjects. Among these results, the iTMT Velocity had the largest effect sizes to distinguish between the 3 groups (Fig. 4). The iTMT Velocity was $6.31 \pm 0.98$ unit/s in the older robust group and was significantly decreased on average by $10 \%$ in the non-robust group $(d=0.62, p=$ $0.025)$. When compared with the older groups, a significantly higher iTMT Velocity of $7.30 \pm 1.13$ unit/s was observed in the young group (Fig. 4). Using the iTMT Velocity, we further compared between the pre-frail and frail subjects. Results suggested that the iTMT Velocity was able to discriminate pre-frail and frail with very large effect size $(d=1.50, p=0.002)$.

Figure 5 demonstrated the correlation between the iTMT and over ground gait test at habitual speed. A significant correlation was observed between the iTMT Velocity and gait speed $(r=0.684, p<0.001)$.

In the univariate regression analysis, 4 variables were significantly associated with frailty: age, gait speed, iTMT Velocity, and iTMT Power (Table 4). In Model 1, only age remained in the model. In Model 2, only age and gait speed remained in the model. Similarly, in Model 3, age and iTMT Velocity remained in the model. The receiver operating characteristic curves for the 3 models were displayed in Figure 6. The AUC for Model 1 was 0.708, with a sensitivity of $78.4 \%$ and specificity of $45.8 \%$ for predicting frailty. The AUC for Model 2 was 0.795 , with a sensitivity of $79.3 \%$ and specificity of $61.9 \%$ for predicting frailty. The highest AUC (0.834) was obtained by Model 3 , with a sensitivity of $82.8 \%$ and specificity of $66.7 \%$ for distinguishing non-robust cases. 


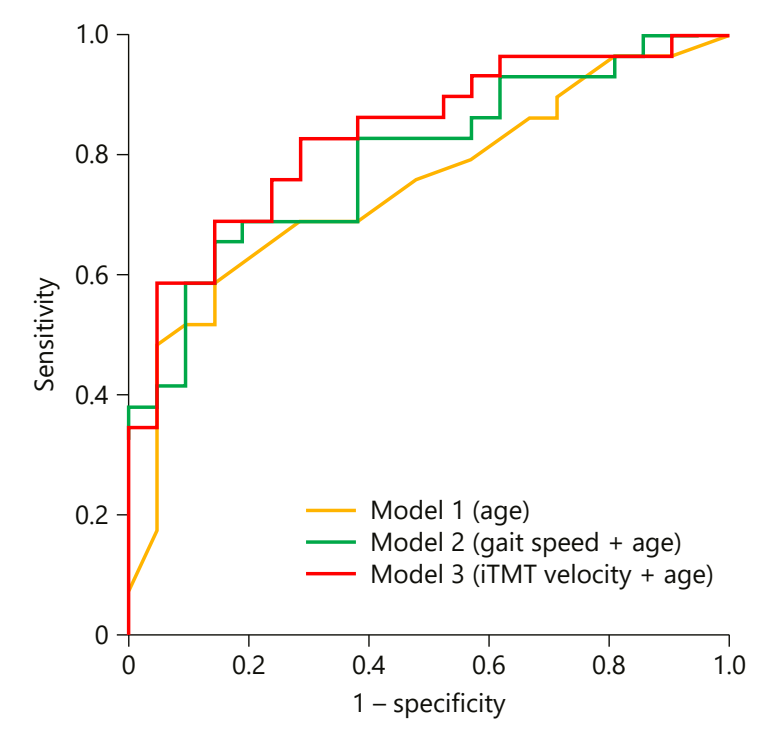

Fig. 6. ROCs of different models for predicting frailty: Model 1 used "age" (AUC = 0.708), Model 2 used a combination of "gait speed" and "age" (AUC $=0.795)$, and Model 3 used a combination of the "instrumented trail-making task (iTMT) Velocity" and “age” (AUC = 0.834).

\section{Discussion}

This study demonstrated the feasibility and validity of an innovative iTMT to quantify physical frailty phenotypes. In addition, it examined the discriminative validity of the iTMT to distinguish between robust individuals and those with frailty (pre-frail or frail), as determined by the FFC. Results demonstrated that the iTMT-derived parameters can identify the presence and absence of key physical frailty phenotypes (slowness, weakness, exhaustion, and inactivity) with largeto-very-large effect sizes $(d=0.90-1.40)$. In addition, the iTMT Velocity could discriminate between robust and pre-frail or frail individuals with medium effect size $(d=0.62)$. Comparing with other iTMT-derived parameters, the iTMT Velocity had the largest power to distinguish different frailty stages, as determined by the FFC. This finding confirmed previous studies demonstrating that gait speed is the most important indicator of the frailty syndrome [27].

Key advantages of the iTMT platform include its practicality for a busy setting (unlike the FFC, it does not require a walking test to determine physical frailty), relatively low cost (as it only needs a single inertial sensor and a standard computer), time efficiency (as the whole test takes less than $5 \mathrm{~min}$ ), and objectivity (as a fully automated test to determine frailty).

From the ease of administration standpoint, this study demonstrated that the interactive interface of the iTMT and computerized instructions provided to the subjects were clear enough to administer the test with minimal supervision from the examiner. This allows reducing the potential bias related to the examiner's experience. It may also provide the opportunity to administer the test by non-expert staff and those with minimum training, making the administration of the test feasible, irrespective of setting, including in-clinic and in-home. Furthermore, it may facilitate the integration of the test for telemedicine applications, in which the individual could perform the test while standing in front of a telemedicine tablet or screen. More importantly, all subjects, including frail ones and those who needed walking assistance, can complete the test. In our study, $16 \%$ of subjects were unable to walk without assistance or walking aid; and $23 \%$ had history of falls. For these individuals, gait assessment might be challenging or difficult to safely administer. No adverse event or difficulty was observed in the current study for the iTMT test.

From the time-efficacy standpoint, our results suggested that the completion time of the iTMT test was, on average, $125 \pm 85 \mathrm{~s}$; and subject preparation and sensor setup were less than $2 \mathrm{~min}$, making the whole operation less than $5 \mathrm{~min}$. This adds advantages when comparing to the FFC assessment, which usually takes 15-20 min [28], thus making the iTMT suitable and potentially cost effective for busy clinics.

Previous studies $[27,29]$ have often evaluated slowness by recording an individual's walking speed from one point to another. For the iTMT, we use the subject's ankle-rotation velocity to determine slowness, while standing in front of a computer with no need of a walking test. In addition, subjects can hold a chair or table placed in front of them to avoid falling or to maintain balance if needed. This has superior benefits compared to the FFC walking test, since administrating the FFC walking test in a busy clinical setting is often impractical; could be hazardous, depending on the subject's risk of falling; and may be biased by the type of footwear, walking accessories (e.g., cane and walker), and walking test condition [20,30]. On the other hand, the iTMT test requires devices, including a single inertial sensor and a standard computer, which may add to the cost when comparing to the conventional FFC walking test. We believe this tradeoff may still be acceptable, considering the time saved by approximately a factor of 3 when comparing to the FFC. In addition, the iTMT test does not need walking space, which makes it 
more convenient than conventional physical frailty assessments based on the FFC walking analysis. Although there is a significant correlation between the iTMT Velocity and gait speed, it should be recognized that the iTMT may not provide all useful features from gait tests for all clinical applications. However, it may be an acceptable surrogate to determine slowness and frailty stages.

Our results suggest that the iTMT Velocity has the largest effect size to identify the presence of slowness phenotype, as determined by the FFC. This confirms another finding of the current study, in which we demonstrated that the iTMT Velocity has significant correlation with gait speed. The finding is also consistent with other studies, which have demonstrated that speed of joint rotation can be used as a surrogate of gait speed. For example, Toosizadeh et al. [31] have demonstrated that speed of elbow rotation during a repetitive 20-s elbow flexion-extension task is correlated with gait speed, which can determine slowness and frailty in older adults. We found the percentage of decline in maximum ankle-rotation velocity (iTMT Exhaustion) has the largest effect size to identify the presence of exhaustion phenotype in the FFC. This result is supported by the finding in a study by Toosizadeh et al. [31] that the speed reduction of elbow flexion-extension in $20 \mathrm{~s}$ provides a quantitative measure of the exhaustion frailty phenotype. We found the power of ankle-rotation (iTMT Weakness) can identify the presence of weakness. This finding is consistent with previous studies demonstrating that power generated during joint rotation is consistent with grip strength and the weakness phenotype in older adults $[25,31]$. Among the iTMT parameters, the iTMT Variability had the largest effect size to identify inactivity phenotype. According to the cycle of frailty suggested by Fried et al. [32], inactivity is a marker of reduction in total energy expenditure in frail people. We speculate that low total energy expenditure could also be explained by poor motor efficiency and high motor variability. These can be measurable by wearable sensors and can be quantified by the iTMT Variability. This is supported by prior studies, which suggest that large gait variability is associated with increasing in metabolic cost and decreasing physical activity level $[33,34]$. In this study, we did not find any iTMT-derived parameter that reliably identifies the presence of the shrinking phenotype. This may be due to insufficient power, as our sample included only 4 cases with the presence of shrinking phenotype.

The results of regression analysis suggested that age alone (Model 1) has poor specificity to distinguish frailty. When adding gait speed into the model (Model 2), the specificity was increased from 46 to $62 \%$ with modest in-

Using Wearable and Virtual Reality to Determine Frailty crease in sensitivity from 78 to $79 \%$. When replacing gait speed with the iTMT Velocity (Model 3), both sensitivity and specificity improved and reached 83 and $67 \%$ respectively. Together, we concluded that the iTMT could be a good surrogate for the FFC walking test to determine frailty status independent of age. But future studies are needed to demonstrate whether the proposed model is clinically meaningful to predict health outcomes in geriatric populations.

In our previous studies [16, 17], we have demonstrated that the iTMT is able to assess cognitive impairment in older adults. The iTMT test has significant agreement $(r>0.65)$ with conventional cognitive screening tools, including Trail Making Test A\&B and Montreal Cognitive Assessment. Combined with the results of this study, we may speculate that the iTMT enables assessing "cognitive frailty" (frailty together with cognitive impairment) [35], which has been shown to be a strong and independent predictor of cognitive decline over time [36, 37]. If this hypothesis is confirmed by future studies, the iTMT may have the potential to detect the subtle progression of cognitive decline over time, which in turn may facilitate the early intervention and prevention of further cognitive and functional decline [38].

\section{Limitations and Future Directions}

One limitation of this study is that our subjects were recruited from specialized outpatient clinics instead of older adults dwelling in the community. Thus, our sample may not represent the general older population. The observation needs to be confirmed in a larger study representing the general older population.

In this study, the number of frail subjects was very small ( $n=8,13 \%$ of older participants in our sample). This number is in line with the prevalence of frailty in the general population, which was estimated to be $12 \%[1,39]$. However, for the purpose of between-group comparison, 8 subjects were considered underpowered for a fair comparison. Thus, we merged pre-frail and frail subjects into a single group. However, when we compared frail and prefrail groups, the iTMT Velocity can distinguish between groups, with a very large effect size $(d=1.50)$. According to this effect size, to achieve a minimum power of $80 \%$, a minimum sample size of 9 subjects per group is required to observe a statistical significance of $5 \%$ or lower, using 2 -tailed independent-sample comparisons.

In this study, we did not adjust for age because frailty is a geriatric symptom; and the likelihood of frailty increases with age. In addition, our reference to determine different frailty stages was the FFC, which has considered 
age to determine cutoff points for the presence and absence of frailty phenotypes. The purpose of this study was to evaluate the feasibility and ability of using the iTMT to quantify frailty phenotypes and distinguish different frailty stages, as determined by the FFC. Thus, considering that our gold standard has already been adjusted by age, further adjustment was determined to be unnecessary for the purpose of this study. Furthermore, when age and the iTMT-derived parameters were used in a multivariate model, the iTMT Velocity remained a significant predictor of non-robustness, independent of age. Another study is needed to demonstrate that the iTMT, irrespective of age, is also able to predict adverse health outcomes in the geriatric population.

We have demonstrated that, when gait speed was replaced by the iTMT Velocity, the power of the model to distinguish frailty status was improved. However, we did not explore other gait parameters and other gait test conditions (e.g., dual task test), which may help to improve the accuracy of a gait-based model to determine frailty status [27]. Thus, this study cannot conclude that the iTMT is superior than the gait test to determine physical frailty but rather that it is more practical in some circumstances in which the administration of gait testing is cumbersome or impractical.

In this study, we demonstrated that the iTMT has a significant correlation with the continuous frailty phenotype variable, such as gait speed. However, a continuous scale to describe frailty stages similar to the Rockwood Frailty index has not been developed because of the selection of the FFC as our gold standard. Another study is required to develop a continuous frailty model similar to a validated frailty meter with a continuous scale $[25,40]$. We anticipate that a continuous frailty index may be more sensitive to change than a categorical frailty model, as suggested in previous studies $[25,40]$. In addition, we anticipate that measurement of both physical frailty and cognitive function, as offered by the iTMT, would be more sensitive to track the changes in life independency and pathway to dementia, as suggested by literature on cognitive decline and frailty $[36,37]$. These hypotheses should be validated in future studies.

\section{Conclusions}

To our knowledge, the iTMT is the first platform enabling the simultaneous and objective measurements of both physical frailty and cognitive performance in the geriatric population. Other key advantages of the iTMT platform are its objectivity, time efficiency, and ability to objectively quantify different frailty phenotypes such as slowness, exhaustion, weakness, and inactivity, with a practical test protocol that does not need self-report or conventional FFC walking examination. Future studies are required to confirm the observation in a larger sample, as well as examine the ability of the iTMT to track changes in physical frailty over time, its sensitivity to intervention, and its ability to predict post-intervention adverse outcomes.

\section{Acknowledgments}

Partial support was provided by the National Institutes of Health/National Institute on Aging (award number R41AG058295) and National Institutes of Health/National Cancer Institute (award number 1R21CA190933-01A1), and Baylor College of Medicine, Michael E. DeBakey Department of Surgery. It was also partly the result of use of resources and facilities at the Houston VA HSR\&D Center for Innovations in Quality, Effectiveness and Safety (CIN13-413). The content is solely the responsibility of the authors and does not necessarily represent the official views of sponsors. We thank Ana Enriquez, Ivan Marin, and Noreen Siddiqi for assisting with data collection and coordination of this research study between involved key investigators.

\section{Disclosure Statement}

The iTMT is protected by a patent (pending). H.Z., J.R., M.K., and B.N. are listed as co-inventors. However, they do not claim any financial conflict of interest relevant to this study.

\section{References}

1 Fried LP, et al: Frailty in older adults: evidence for a phenotype. J Gerontol A Biol Sci Med Sci 2001;56:M146-M156.

2 Morley JE: Diabetes, sarcopenia, and frailty. Clin Geriatr Med 2008;24:455-469, vi.

3 Janssen I, et al: Skeletal muscle cutpoints associated with elevated physical disability risk in older men and women. Am J Epidemiol 2004;159:413-421.
4 Makary MA, et al: Frailty as a predictor of surgical outcomes in older patients. J Am Coll Surg 2010;210:901-908.

5 Mohler MJ, et al: The Frailty syndrome: clinical measurements and basic underpinnings in humans and animals. Exp Gerontol 2014;54: 6-13.
Zhou/Razjouyan/Halder/Naik/Kunik/ Najafi 
6 Podsiadlo D, Richardson S: The timed "up \& go": a test of basic functional mobility for frail elderly persons. J Am Geriatr Soc 1991;39: 142-148.

7 Goldstein JP, Andrew MK, Travers A: Frailty in older adults using pre-hospital care and the emergency department: a narrative review. Can Geriatr J 2012;15:16-22.

8 Buchman A, et al: Change in frailty and risk of death in older persons. Exp Aging Res 2009; 35:61-82.

9 da Câmara SMA, et al: Using the short physical performance battery to screen for frailty in young-old adults with distinct socioeconomic conditions. Geriatr Gerontol Int 2013;13: 421-428.

10 Verghese J, Xue X: Identifying frailty in high functioning older adults with normal mobility. Age Ageing 2010;39:382-385.

11 Goel R, et al: Assessing somatosensory utilization during unipedal postural control. Front Syst Neurosci 2017;11:21.

12 Wang C, et al: Low-power fall detector using triaxial accelerometry and barometric pressure sensing. IEEE Trans Industr Inform 2016;12:2302-2311.

13 Razjouyan J, et al: Wearable sensors and the assessment of frailty among vulnerable older adults: an observational cohort study. Sensors (Basel) 2018;18:pii:E1336.

14 Zahiri M, et al: Design and evaluation of a portable laparoscopic training system using virtual reality. J Med Device 2017;11:011002.

15 Nguyen $\mathrm{H}$, et al: Using inertial sensors to automatically detect and segment activities of daily living in people with Parkinson's disease. IEEE Trans Neural Syst Rehabil Eng 2018;26:197-204.

16 Zhou H, et al: Instrumented trail-making task to differentiate persons with no cognitive impairment, amnestic mild cognitive impairment, and Alzheimer disease: a proof of concept study. Gerontology 2017;63:189-200.

17 Zhou H, et al: Motor planning error: toward measuring cognitive frailty in older adults using wearables. Sensors (Basel) 2018;18: pii:E926.
18 Delbaere K, et al: The falls efficacy scale international (FES-I). A comprehensive longitudinal validation study. Age Ageing 2010;39: 210-216.

19 Weissman MM, et al: Assessing depressive symptoms in five psychiatric populations: a validation study. Am J Epidemiol 1977;106: 203-214.

20 Najafi B, et al: Does walking strategy in older people change as a function of walking distance? Gait Posture 2009;29:261-266.

21 Grewal G, et al: Virtualizing the assessment: a novel pragmatic paradigm to evaluate lower extremity joint perception in diabetes. Gerontology 2012;58:463-471.

22 Najafi B, et al: Assessing postural control and postural control strategy in diabetes patients using innovative and wearable technology. J Diabetes Sci Technol 2010;4:780-791.

23 Najafi B, et al: Estimation of center of mass trajectory using wearable sensors during golf swing. J Sports Sci Med 2015;14:354-363.

24 Grewal GS, et al: Sensor-based interactive balance training with visual joint movement feedback for improving postural stability in diabetics with peripheral neuropathy: a randomized controlled trial. Gerontology 2015; 61:567-574.

25 Lee $\mathrm{H}$, et al: Toward using a smartwatch to monitor frailty in a hospital setting: using a single wrist-wearable sensor to assess frailty in bedbound inpatients. Gerontology 2018; 64:389-400.

26 Cohen J: Statistical power analysis for the behavioral sciences (ed 2). Hillsdale, Erlbaum, 1988.

27 Schwenk M, et al: Frailty and technology: a systematic review of gait analysis in those with frailty. Gerontology 2014;60:79-89.

28 Gary R: Evaluation of frailty in older adults with cardiovascular disease: incorporating physical performance measures. J Cardiovasc Nurs 2012;27:120-131.
29 Schwenk M, et al: Wearable sensor-based inhome assessment of gait, balance, and physical activity for discrimination of frailty status: baseline results of the Arizona frailty cohort study. Gerontology 2015;61:258-67.

30 Najafi B, et al: The impact of footwear and walking distance on gait stability in diabetic patients with peripheral neuropathy. J Am Podiatr Med Assoc 2013;103:165-173.

31 Toosizadeh N, Mohler J, Najafi B: Assessing upper extremity motion: an innovative method to identify frailty. J Am Geriatr Soc 2015; 63:1181-1186.

32 Fried LP, Walston J. Hazzard WR, Blass JP, Ettinger WH, Jr, Halter JB, Ouslander J: Principles of Geriatric Medicine and Gerontology (ed 4). New York, McGraw Hill, 1998, pp 1387-1402.

33 O'Connor SM, Xu HZ, Kuo AD: Energetic cost of walking with increased step variability. Gait Posture 2012;36:102-107.

34 Montero-Odasso M, et al: Gait variability is associated with frailty in community-dwelling older adults. J Gerontol A Biol Sci Med Sci 2011;66:568-576

35 Shimada $\mathrm{H}$, et al: Cognitive frailty and incidence of dementia in older persons. J Prev Alzheimer's Dis 2018;5:42-48.

36 Kelaiditi E, et al: Cognitive frailty: rational and definition from an (I.A.N.A./I.A.G.G.) international consensus group. J Nutr Health Aging 2013;17:726-734.

37 Ruan Q, et al: Cognitive frailty, a novel target for the prevention of elderly dependency. Ageing Res Rev 2015;20:1-10.

38 Dierckx E, et al: Mild cognitive impairment: what's in a name? Gerontology 2007;53:2835.

39 Buckinx F, et al: Burden of frailty in the elderly population: perspectives for a public health challenge. Arch Public Health 2015;73: 19.

40 Joseph B, et al: Upper-extremity function predicts adverse health outcomes among older adults hospitalized for ground-level falls. Gerontology 2017;63:299-307. 